\title{
[ERRATUM] "SEJONG OPEN CLUSTER SURVEY (SOS). 0. TARGET SELECTION AND DATA ANALYSIS"
}

Hwankyung Sung ${ }^{1}$, Beomdu Lim ${ }^{1}$, Michael S. Bessell ${ }^{2}$, Jinyoung S. Kim$^{3}$, Hyeonoh Hur ${ }^{1}$, Moo-Young Chun ${ }^{4}$, AND BYEONG-GON PARK ${ }^{4}$

${ }^{1}$ Department of Astronomy and Space Science, Sejong University, Neungdong-ro 209, Gwangjin-gu, Seoul 143-747, Korea

E-mail : sungh@sejong.ac.kr

${ }^{2}$ Research School of Astronomy and Astrophysics, Australian National University, MSO, Cotter Road, Weston, ACT 2611, Australia

E-mail : bessell@mso.anu.edu.au

${ }^{3}$ Steward Observatory, University of Arizona, 933 N. Cherry Ave., Tucson, AZ 85721-0065, USA

E-mail : serena@as.arizona.edu

${ }^{4}$ Korea Astronomy and Space Science Institute, Daedeok-daero 776, Yuseong-gu, Daejeon 305-348, Korea

E-mail : mychun,bgpark@kasi.re.kr

(Received September 17, 2013; Accepted September 17, 2013)

Eq. 9 had a typographical error. The correct expression of the equation isas follows. The authors sincerely regret this error.

$$
Q^{\prime} \equiv(U-B)-0.72(B-V)-0.025 E(B-V)^{2}
$$

